\title{
TRIAGE DOWN AND DIRTY
}

\section{DEFINITION OF TRIAGE}

Triage is a sorting process used to identify the sickest patients or those at greatest risk of demise so immediate medical needs can be rapidly addressed. Triage comes from the French verb "trier," which means "to sort."

\section{GOALS OF TRIAGE}

The goal of triage is to determine:

1. How sick or injured is the patient? What is this patient's potential for demise?

2. How quickly do interventions, care and treatment need to be delivered?

3. What treatment/waiting area of the department is most appropriate for the patient (main ED vs. Fast Track, internal waiting room, etc.)?

In this book, the focus is on identifying patients who require immediate or rapid intervention and care. 


\section{FIVE-LEVEL TRIAGE SCALE}

Several valid and reliable five-level triage systems exist, but no specific triage system is referred to in this guide.

Understanding a five-level triage scale is a complex process requiring formalized triage education followed by hands-on training with a preceptor. A combination of education, experience, and critical thinking, in conjunction with being a detective in questioning and assessment, plays a role in getting to the most accurate triage level. Terms associated with triage levels within this book refer to a five-level triage system only and include:

\section{Level 1}

- The patient is a resuscitation.

\section{Level 2}

- The patient has an emergent condition.

\section{Level 3}

- The patient has an urgent condition.

\section{Level 4}

- The patient has a less-urgent condition.

\section{Level 5}

- The patient has a non-urgent condition.

Source: Gilboy, N., Tanabe, T., Travers, D., \& Rosenau, A. M. (2011, November). Emergency Severity Index (ESI): A triage tool for emergency department care, version 4. Implementation Handbook 2012 Edition. AHRQ Publication No. 12-0014. Rockville: MD. Agency for Healthcare Research and Quality. 


\section{THE PATIENT ARRIVAL}

A systematic approach to triage helps rapidly identify the sickest patients and get them to the right care.

The initial assessment of the patient arrival should involve the following:

\section{Primary Survey/Across the Room Assessment or the "Quick Look"}

- Evaluate the patient's A-B-C-D-E upon arrival.

- Act on any significant A-B-C-D-E impairments.

- Consider C-spine immobilization for any known traumatic injuries.

- If immediately identified as needing life-saving or rapid treatment, move patient straight to the treatment area.

\section{Rapid Triage Assessment (takes $<60-90$ seconds)}

- Obtain the chief complaint, name, date of birth.

- Consider the need for patient decontamination.

- Think Red Flag Findings and assess for them (Red Flags are listed in each body system section).

- Think screening for trauma, STEMI, stroke, and sepsis; initiate per protocol at triage! Time is critical and outcomes improve if timely treatment is initiated upon identification.

- Consider if the patient needs a stat ECG per protocol; think ECG in less than 10 minutes of arrival at facility.

- Continue to evaluate for A-B-C-D-E abnormalities and c-spine immobilization as information is available.

NOTE: A-B-C-D-E stands for Airway - Breathing - Circulation - Disability - Expose/Environment 


\section{RED FLAG FINDINGS-HIGH-RISK PRESENTATIONS}

- While obtaining the chief complaint, the nurse asks questions to determine the patient's potential for a permanent impairment or demise.

- If the patient has a red flag finding, you may have a life-threatening or high-risk patient presentation; immediate or rapid medical care (in most cases) is needed.

- Red flag findings always require critical thinking and clinical correlation with assessment.

\section{EMERGENCY KEY TIPS}

- Key tips cover the most common concerns for the body system and apply to most of the chief complaints listed (unless otherwise stated).

- Other chapters to consider are addressed (e.g., do not assume arm pain is a musculoskeletal issue).

- Orders to anticipate are listed. Facility ATPs might include these orders.

\section{ED METRICS}

- Arrival Time: The time the patient enters the facility seeking care.

- Door-to-Doctor Time: The time interval in minutes between arrival time and patient consultation time by a physician/provider.

- Door-to-Discharge Time: The time interval in minutes between arrival at the facility and discharge.

- ED LOS: The time the patient spent in the ED from the arrival time until discharge. 


\section{PRIORITY SETTING}

When considering what patient presentations may take precedence over others, refer to Table 1.1: Priority Setting at Triage.

\section{Table 1.1: Priority Setting at Triage}

\begin{tabular}{|l|l|}
\hline \multicolumn{1}{|c|}{ FIRST PRIORITY } & \multicolumn{1}{c|}{ SECOND PRIORITY } \\
\hline Severe A-B-C-D-E impairment & Minor A-B-C-D-E impairment \\
\hline Life-threatening situation & Limb-threatening situation \\
\hline Cannot rule out worst-case scenario & Can rule out worst-case scenario \\
\hline Acute onset & $\begin{array}{l}\text { Chronic conditions (if condition has changed, consider } \\
\text { increasing prioritization) }\end{array}$ \\
\hline Systemic issues & Localized issues \\
\hline High-risk factors (e.g., comorbidities) & No high-risk factors \\
\hline
\end{tabular}

